\title{
Aspiration during Rapid Sequence Induction: Prevalence and Risk Factors
}

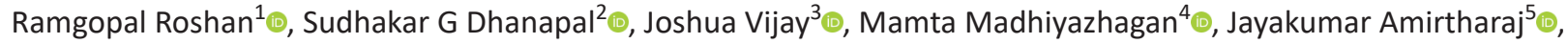 \\ Priya Ganesan $^{6}{ }^{\circ}$, Kundavaram PP Abhilash $^{7}{ }^{\circ}$
}

\begin{abstract}
Background: Securing definitive airway with minimal complications is a challenging task for high-volume emergency departments (ED) that deal with patients with compromised airway.

Materials and methods:We conducted a prospective observational study between September 2019 and March 2020. Cohort of adults presenting to the ED requiring rapid sequence induction (RSI) were recruited to determine the prevalence and risk factors for the development of aspiration pneumonia(AP) in patients intubated in the ED.

Results: During the study period, a total of 154 patients with a mean age of 44.5 years required RSI in the ED. Male (61\%) predominance was noted among the study cohorts. We did not find any association between RSI performed in the ED and the risk of developing AP. The first attempt success rate of RSI was 76.7\%, and 33(21.4\%) patients had immediate adverse events following RSI. Rescue intubation was required for $11(7.1 \%)$ patients. The prevalence of AP following RSI in the ED was $13.4 \%$. Endotracheal tube (ET) aspirate pepsin was positive in $45(29.2 \%)$ samples collected. The ET aspirate pepsin assay had low sensitivity (44.44\%), specificity (73.53\%), positive predictive value (18\%), and negative predictive value (91\%) in predicting the occurrence of AP. On multivariate logistic regression analysis, male gender (AOR: $7.29,95 \% \mathrm{Cl}: 1.51-35.03, p=0.013$ ) and diabetes mellitus (AOR: $3.75,95 \% \mathrm{Cl}: 1.23-11.51, p=0.02$ ) were found to be independent risk factors for developing AP.

Conclusion: We identified male gender and diabetes mellitus to be independent predictors of risk of developing AP after RSI in the ED. ET aspirate pepsin levels proved to be neither sensitive nor specific in the diagnosis of AP.

Keywords: Aspiration, Aspiration pneumonia, Emergency department, Endotracheal intubation, Pepsin.

Indian Journal of Critical Care Medicine (2021): 10.5005/jp-journals-10071-23714
\end{abstract}

\section{INTRODUCTION}

The emergency department (ED) is considered as the face of the hospital and first line of contact to health care for the critically ill. ${ }^{1}$ The success of resuscitation of critically ill patients reflects the standards of the ED. Endotracheal intubation is considered as the gold standard airway management in the ED for patient requiring definitive airway. ${ }^{2}$ It is one of the most commonly performed invasive procedures in the ED. Due to limited time for preparation, unstable condition of the patient, and urgency of the situation, it is always considered as a high-risk procedure. ${ }^{3} \mathrm{~A} 12-26 \%$ intubationrelated adverse events have been reported in the literature. ${ }^{4-6} \mathrm{RSI}$ includes the use of an induction agent, followed by a paralytic agent to achieve optimal conditions for intubation. This drug regimen should produce sedation and neuromuscular paralysis, maintain hemodynamic stability, and prevent vomiting. ${ }^{7}$ Drugs with rapid onset, short duration of action, and minimal adverse effects are usually administered.

Aspiration is defined as the inhalation of either oral, pharyngeal, or gastric contents into the lower airways. ${ }^{8}$ This can lead to a number of syndromes based on the quantity and nature of the aspirated material, the frequency of aspiration, and the host factors that predispose the patient to aspiration and modify the response. ${ }^{8}$ The epidemiological study on AP is complicated due to lack of specific and sensitive marker to diagnose AP and difficulty to distinguish between aspiration syndromes(aspiration pneumonitis and AP). ${ }^{9}$ The diagnosis of AP is made when a patient at risk for aspiration is found to
1-4,6,7 Department of Emergency Medicine, Christian Medical College, Vellore, Tamil Nadu, India

${ }^{5}$ Department of Clinical Biochemistry, Christian Medical College, Vellore, Tamil Nadu, India

Corresponding Author: Ramgopal Roshan, Department of Emergency Medicine, Christian Medical College, Vellore, Tamil Nadu, India, Phone: +919840963003, e-mail: roshan.rajaram93@gmail.com

How to cite this article: Roshan R, Sudhakar GD, Vijay J, Mamta M, Amirtharaj J, Priya G, et al. Aspiration during Rapid Sequence Induction: Prevalence and Risk Factors. Indian J Crit Care Med 2021;25(2):140-145. Source of support: Fluid Research Grant of Christian Medical College, Vellore, Tamil Nadu, India

Conflict of interest: None

have a radiographic evidence of an infiltrate in a characteristic bronchopulmonary segment. ${ }^{10}$ The course of the disease is similar to that of a typical community-acquired pneumonia, but has a higher risk of developing cavitation and abscess formation, if left unrecognized or untreated. ${ }^{10}$ Pepsin is the major proteinase in the stomach secreted by the chief cells. It is normally absent in the respiratory tract. So the presence of pepsin in the respiratory tract implies that aspiration had occurred. Also, the ET aspirate will contain gastric contents if they were aspirated and this can be used to demonstrate the presence of pepsin. ${ }^{11}$ In general, there is a higher risk of aspiration and developing other complications

(c) Jaypee Brothers Medical Publishers. 2021 Open Access This article is distributed under the terms of the Creative Commons Attribution 4.0 International License (https://creativecommons.org/licenses/by-nc/4.0/), which permits unrestricted use, distribution, and non-commercial reproduction in any medium, provided you give appropriate credit to the original author(s) and the source, provide a link to the Creative Commons license, and indicate if changes were made. The Creative Commons Public Domain Dedication waiver (http://creativecommons.org/publicdomain/zero/1.0/) applies to the data made available in this article, unless otherwise stated. 
among patients intubated in the ED compared to those in intensive care units (ICUs) and operating rooms (ORs). ${ }^{12}$ Hence, we conducted this study to determine the prevalence and risk factors for the development of AP in patients who undergo RSI in the ED and to determine the sensitivity and specificity of pepsin to aid the diagnosis of AP.

\section{Materials and Methods Study Design \\ A prospective observational study.}

\section{Study Setting}

We conducted this study in the ED of a tertiary care hospital in South India, between September 2019 and March 2020. Our ED is a 49-bed department and is one of the busiest departments in whole country with an average caseload of 250 patients per day. We have a dedicated 6 bedded resuscitation rooms (RRs), exclusively for the management of patients who present with airway and hemodynamic compromise.

\section{Participants}

We screened all patients presenting to the ED requiring RSI during the 7-month study period.

\section{Inclusion Criteria}

All participants aged more than 18 years requiring $\mathrm{RSI}$ and were admitted as in-patients.

\section{Exclusion Criteria}

Patients with preexisting pneumonia, patients with direct lung injury (secondary to trauma), and those not willing to consent were excluded.

\section{Variables}

Variables, such as comorbidities, presenting complaints, indication for intubation, vital signs, drugs used as premedications, laryngoscopy blade, operator expertise, number of attempts, and Cormack-Lehane grading were compared to the risk of developing AP.

\section{Outcome Variable}

The outcome variables were defined as follows:

Aspiration pneumonia-Any of the following in patients without a diagnosis of community-acquired or healthcareassociated pneumonia, or aspiration prior to intubation: pathogenic growth in sputum culture, unexplained hypoxemia, or radiographic evidence of pneumonia in the first 48 hours after intubation. ${ }^{13}$

Ventilator-associated pneumonia-Radiographic evidence of pneumonia on chest x-ray, pathogenic growth in sputum culture, or unexplained hypoxia that occurred between $>48$ hours after intubation and $<48$ hours after extubation. ${ }^{13}$

\section{Bias}

All consecutive patients requiring RSI were screened and those consented to take part in the study were recruited.

Sample size: Based on a previous study, the incidence of AP was estimated to be $13 \%$, with a precision of $5 \%$ and confidence level of $95 \%$, and the sample size was calculated to be $174 .{ }^{14}$

\section{Sample Collection}

Tracheal aspirates were obtained using a standard Lueken's trap as soon as endotracheal tube position was confirmed using visualization, auscultation, and end-tidal $\mathrm{CO}_{2}$. If no aspirate could be obtained, 3-5 $\mathrm{mL}$ of normal saline solution was instilled into the endotracheal tube. Each patient was ventilated for several breaths, and the sample was then collected. Tracheal aspirates were collected and marked with a study number and were sent to the biochemistry laboratory for assay.

\section{Laboratory Test}

The tracheal samples that were received in the laboratory were centrifuged at $2000 \mathrm{rpm}$ for 20 minutes, and an aliquot was stored frozen at $-20^{\circ} \mathrm{C}$ until analysis. The aliquot was then thawed and used for analysis by enzyme-linked immunosorbent assay (ELISA), from Sincere Biotech Co. Ltd., China; Catalog No: E13651380 was used for the in vitro quantitative determination of human pepsin antigen from tracheal aspirates. Six different standard concentrations 0 , $7.5,15,30,60$, and $90 \mathrm{ng} / \mathrm{mL}$ were prepared. The standards and tracheal samples $(50 \mu \mathrm{L})$ whose pepsin concentration is to be estimated were added to the antibody-coated wells. The plate with the standards and samples was incubated for 30 minutes at $37^{\circ} \mathrm{C}$ so as to form an antibody-antigen complex. Following the wash, $50 \mu \mathrm{L}$ of horseradish peroxidase (HRP) conjugate was added and the plate was incubated for 30 minutes at $37^{\circ} \mathrm{C}$ so as to form antibody-antigen-enzyme conjugate complex. Following the wash, $100 \mu \mathrm{L}$ of tetramethylbenzidine (TMB) chromogen substrate was added and incubated for 15 minutes at $37^{\circ} \mathrm{C}$ where the TMB substrate becomes blue in color with the HRP enzymecatalyzed reaction. This reaction was terminated by the addition of a sulfuric acid solution, and the color change (yellow) was measured spectrophotometrically at a wavelength of $450 \mathrm{~nm}$. The concentration of human pepsin antigen in the samples was then determined by comparing the absorbance of the samples to the standard curve.

\section{Statistical Analysis}

All continuous variables were expressed with mean and standard deviation, and categorical variables were expressed as frequencies and percentages. The data were analyzed using Statistical Package for the Social Sciences (SPSS) for Windows software released 2015, version23.0, Armonk, New York, USA. Categorical variables for the risk of developing AP were analyzed using chi-square test or Fisher's exact test. Odds ratio and their $95 \%$ confidence intervals were calculated. For all variables, a two-sided $p$-value less than 0.05 was considered statistically significant. A logistic regression analysis was done on variables, which had significance on bivariate analysis.

\section{Ethical Considerations}

Prior to the commencement of the study, approval from the Institutional Review Board (IRB) and Ethical Committee was obtained (IRB Min no: 11919 dated March 6, 2019).

\section{Results}

A total of 47,629 patients presented to our ED during the study period. We screened all 338 patients requiring RSI in the ED, and 184(54\%) patients were excluded as they did not fulfill the inclusion criteria, leaving 154 patients for further analysis (Flowchart 1). 


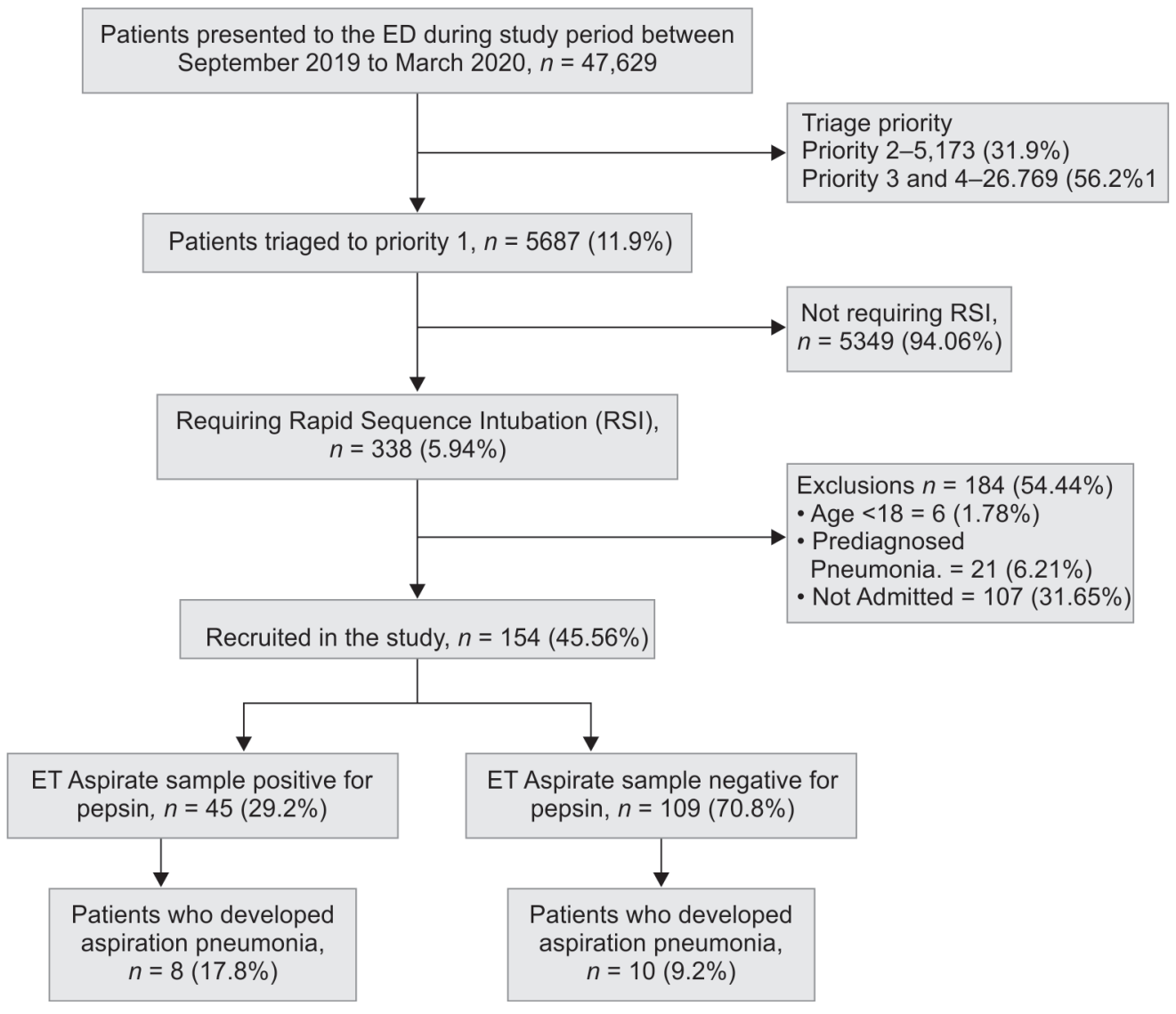

Flowchart 1: STROBE diagram

\section{Characteristics of Study Subjects}

Table 1 shows the baseline characteristics. The mean age was $44.5 \pm 16.04$ years. Our study cohort had a male predominance (61\%).

\section{Intubation Factors and Risk of Developing AP}

Table 2 shows the premedications, laryngoscope, ET used, Cormack-Lehane grading on laryngoscopy, attempts at intubation, experience of the intubator, and the application of cricoid pressure among the study cohort. The mean time taken to perform RSI was 12.74(+9.56) minutes. Eleven (7.1\%) patients required intubation by a rescue intubator. In the pre-intubation period, $14.3 \%$ of patients desaturated, requiring prolonged preoxygenation (Table 3 ). We observed that $15(9.7 \%)$ patients had a witnessed aspiration and 8(5.2\%) patients developed hypotension during the peri-intubation period. Inadvertent placement of ET into the esophagus was observed in $3.9 \%$ of patients (Table 4 ).

\section{Outcome Measures}

Twenty patients died within 48 hours of intubation in the ED. These patients were excluded from the primary outcome measurement. The prevalence of AP following RSI in the ED was $13.4 \%(18 / 134)$. The tracheal aspirate pepsin was positive $(>12.3 \mathrm{ng} / \mathrm{mL})$ in $45(29.2 \%)$ patients. However, only $8(44.44 \%)$ patients who developed AP had positive pepsin levels. We performed an area under receiver operating characteristic (AUROC) curve for determining the sensitivity and specificity of pepsin in predicting the development of
Table 1: Baseline characteristics, $n=154$

\begin{tabular}{ll}
\hline Variable & $n(\%)$ \\
\hline Age, mean (SD), in years & $44.49(16.17)$ \\
Gender & \\
Male & $94(61)$ \\
Female & $60(39)$ \\
Time of arrival & \\
12 am to 8 am & $28(18.2)$ \\
8 am to 4 pm & $50(32.5)$ \\
4 pm to 12 am & $76(49.4)$ \\
Comorbidities & \\
Diabetes mellitus & $45(29.2)$ \\
Hypertension & $43(27.9)$ \\
Chronic kidney disease & $8(5.2)$ \\
Gastroesophageal reflux disease & $2(1.3)$ \\
Pregnancy & $1(0.6)$ \\
Complaints & \\
Breathing difficulty & $87(56.5)$ \\
Altered sensorium & $63(40.9)$ \\
Vomiting & $40(26.0)$ \\
Trauma & $36(23.4)$ \\
Fever & $33(21.4)$ \\
Chest pain & $16(10.4)$ \\
\hline &
\end{tabular}


Table 2: Rapid sequence induction parameters among the study population

\begin{tabular}{|c|c|}
\hline Variable & $n(\%)$ \\
\hline \multicolumn{2}{|l|}{ Induction agent } \\
\hline Ketamine & $53(34.4)$ \\
\hline Midazolam & $49(31.8)$ \\
\hline No drugs & $14(9.1)$ \\
\hline Fentanyl & $5(3.3)$ \\
\hline \multicolumn{2}{|l|}{ Paralyzing agent } \\
\hline Succinylcholine & $101(65.6)$ \\
\hline Rocuronium & $35(22.7)$ \\
\hline No drugs & $16(10.4)$ \\
\hline \multicolumn{2}{|l|}{ Laryngoscope } \\
\hline Direct laryngoscopy & $145(94.2)$ \\
\hline Video laryngoscope & $9(5.8)$ \\
\hline \multicolumn{2}{|l|}{ ET tube used } \\
\hline Size 7.5 & $64(41.6)$ \\
\hline Size 8 & $46(29.9)$ \\
\hline Size 7 & $39(25.3)$ \\
\hline \multicolumn{2}{|l|}{ Cormack-Lehane grading } \\
\hline Grade 1 & $91(59.1)$ \\
\hline Grade 2 & $60(39.0)$ \\
\hline Grade 3 & $2(1.3)$ \\
\hline Grade 4 & $1(0.6)$ \\
\hline \multicolumn{2}{|l|}{ Attempts to intubate } \\
\hline 1 & 118(76.6) \\
\hline 2 & $25(16.2)$ \\
\hline $3+$ & $11(7.1)$ \\
\hline \multicolumn{2}{|l|}{ Experience of personnel } \\
\hline Second-year resident & $59(38.3)$ \\
\hline First-year resident & $50(32.5)$ \\
\hline Third-year resident & $29(18.8)$ \\
\hline Paramedics and interns & $13(8.4)$ \\
\hline Consultant & $3(1.9)$ \\
\hline \multicolumn{2}{|l|}{ Cricoid pressure } \\
\hline No cricoid pressure & $80(51.9)$ \\
\hline Cricoid pressure given & $74(48.1)$ \\
\hline
\end{tabular}

Table 3: Mean time to intubate, rescuer attempts, and adverse events following rapid sequence induction

\begin{tabular}{ll}
\hline Variable & $n(\%)$ \\
\hline Average time to perform RSI (SD), in minutes & $12.74(9.56)$ \\
Requiring rescuer attempt & $11(7.1)$ \\
Adverse events* & \\
Hypoxemia in the pre-intubation period & $22(14.3)$ \\
Peri-intubation vomiting & $15(9.7)$ \\
Post-intubation hypotension & $8(5.2)$ \\
Esophageal intubation & $6(3.9)$ \\
\hline
\end{tabular}

*Adverse events between administration of induction agent and 15 minutes following successful intubation were considered

AP (Fig. 1). The ET aspirate pepsin assay had a sensitivity, specificity, positive predictive value, negative predictive, positive likelihood ratio, and negative likelihood ratio value of $44.44 \%, 73.53 \%, 18 \%$,
91\%, 1.679(Cl: 0.933-3.022), and 0.756(Cl: 0.494-1.156), respectively. Among the study population, 96 (62.4\%) were discharged stable, while $19(12.3 \%)$ left against and in-hospital mortality was observed in $39(25.3 \%)$ patients.

\section{Risk Factors for Developing AP}

We performed a bivariate analysis to determine the risk factors associated with the development of AP. Male gender, diabetes mellitus, and altered sensorium had higher risk of developing $A P$, while using ketamine as induction agent reduces the risk of developing AP. However, on performing multivariate logistic regression analysis, with the above variables, only male gender (AOR: $7.29,95 \% \mathrm{Cl}: 1.52-35.03, p=0.013$ ) and diabetes mellitus (AOR: $3.76,95 \% \mathrm{Cl}: 1.23-11.51, p=0.020$ ) proved to be independent risk factors for the development of AP (Table 4).

\section{Discussion}

Our study showed that the prevalence of AP secondary to endotracheal intubation in the ED was $13.4 \%$. We did not identify any intubation factors that predisposed to the development of AP. ET aspirate pepsin assay had a very low sensitivity and specificity in predicting the occurrence of AP. The occurrence of aspiration of gastric contents following endotracheal intubation in the ED varies from $1 \%$ to $20 \%$ according to known literature. ${ }^{15-20}$ This variation in results may be attributed due to the lack of clear definition of AP. The end points of AP as described in previous studies too were questionable. These included (i) suspicion of aspiration, (ii) witnessed aspiration, (iii) radiographic changes, such as the presence of infiltrates, (iv) sputum cultures, and (v) arterial blood gas analysis, none of which were found to have high sensitivity or specificity for detecting aspiration. ${ }^{8}$

The mean age ( 44.5 years) of our study population was much lesser than the median age in a study done by Lanspa et al. study (77). ${ }^{21}$ The difference in age distribution could be attributed to the fact that in developed countries where the study was done, the life expectancy is longer; hence, the proportion of patients in their study is skewed toward older and more comorbid population. Similar to the findings of Driver et al., none of the factors associated with RSI, such as premedications, paralyzing agents, use of video and direct laryngoscope, the number of attempts to intubate, experience of the intubator, the Cormack-Lehane grading of the vocal cords, and application of cricoid pressure prior to intubation proved to be significant risk factors for the development of AP. ${ }^{13}$ This is perhaps attributable to the study done in a well-established teaching center with supervision, in contrast to majority of hospitals that depend on junior medical officers for managing compromised airways. Though higher use of video laryngoscopy probably resulted in a higher first-attempt success rate, it did not decrease the rate of AP. ${ }^{13}$ The adverse events profile in our study was similar to that seen in other studies. ${ }^{22}$

Data from other studies have revealed that tracheal aspirate pepsin is a reliable marker to diagnose aspiration..$^{11,23-25}$ The qualitative detection of pepsin, by demonstrating pepsin activity on exposure to pepstatin (pepsin inhibitor), proved to be highly sensitive and specific by Ufberg et al. ${ }^{11}$ In our study, quantitative assessment of pepsin from ET aspirate was done and had low sensitivity. Negative pepsin assay in almost half of the patients who developed AP may be due to microaspiration that occurred following ET aspirate collection. 
Aspiration during RSI: Prevalence and Risk Factors

Table 4: Bivariate and multivariate logistic regression analysis of factors associated with the development of aspiration pneumonia

\begin{tabular}{|c|c|c|c|c|c|c|}
\hline & \multicolumn{2}{|c|}{ Variables } & \multicolumn{2}{|c|}{ Bivariate analysis } & \multicolumn{2}{|c|}{ Multivariate analysis } \\
\hline & $\begin{array}{l}\text { Aspiration } \\
\text { pneumonia } \\
n=18(\%)\end{array}$ & $\begin{array}{l}\text { No aspiration } \\
\text { pneumonia } \\
n=136(\%)\end{array}$ & $p$-value & Unadjusted OR(95\%Cl) & $p$-value & Adjusted OR(95\%Cl) \\
\hline Male gender & 16(88.9) & $78(57.4)$ & 0.01 & $5.95(1.32-26.89)$ & 0.013 & $7.29(1.51-35.03)$ \\
\hline Diabetes mellitus & $9(50)$ & $36(26.5)$ & 0.039 & $2.78(1.02-7.55)$ & 0.02 & $3.75(1.23-11.51)$ \\
\hline Altered sensorium & $12(66.7)$ & $51(37.5)$ & 0.018 & $3.33(1.18-9.43)$ & 0.086 & $2.66(0.87-8.19)$ \\
\hline Hypoxia, $\mathrm{SpO}_{2}<94 \%$ & 12(66.6) & $86(63.2)$ & 0.78 & $1.16(0.41-2.20)$ & - & - \\
\hline Midazolam & $9(50)$ & $40(29.4)$ & 0.078 & $2.4(0.89-6.49)$ & - & - \\
\hline Ketamine & $2(11.1)$ & $53(37.5)$ & 0.027 & $0.21(0.04-0.94)$ & 0.061 & $4.57(0.93-22.42)$ \\
\hline Succinylcholine & $12(66.7)$ & $89(65.4)$ & 0.918 & $1.05(0.37-2.99)$ & - & - \\
\hline CL Grade $2^{*}$ & $7(38.9)$ & $53(39.0)$ & 0.44 & $0.6(0.16-2.19)$ & - & - \\
\hline First-attempt intubation & $14(77.8)$ & $104(76.5)$ & 0.767 & $0.77(0.25-2.32)$ & - & - \\
\hline First-year resident & $4(22.2)$ & $46(33.8)$ & 0.323 & $0.56(0.17-1.80)$ & & \\
\hline Cricoid pressure applied & $9(50)$ & $65(47.8)$ & 0.86 & $1.09(0.41-2.92)$ & - & - \\
\hline
\end{tabular}

${ }^{*} \mathrm{CL}$, Cormack-Lehane grading of laryngoscopy view

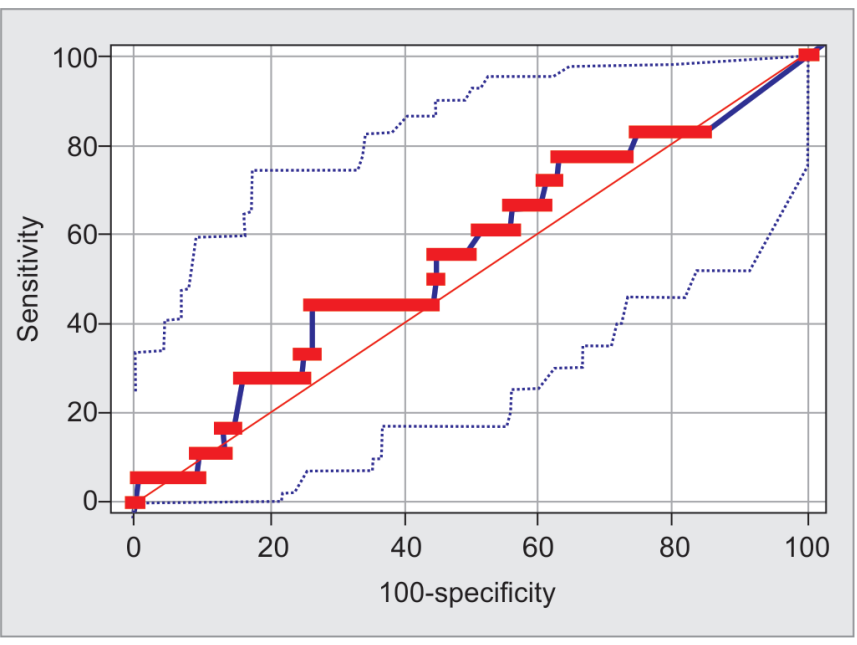

Fig. 1: AUROC curve to assess the sensitivity and specificity of ET aspirate pepsin to predict the development of AP

\section{Strengths}

This was a prospective study with all consecutive patients requiring RSI being enrolled, thereby minimizing the bias. Quantitative assessment of ET aspirate pepsin levels was another strength of our study.

\section{Clinical Implications}

The ET aspirate pepsin assay used in our study was a research kit. There might be variation in results with respect to the research kits.

\section{LiMITATIONS}

In our study, we could not reach the required sample size of 174 , mainly because the Human Pepsin ELISA Kit used could not be imported from China due to travel and transport disruptions during the COVID-19 pandemic.

\section{Conclusion}

From our study, we concluded that male gender and diabetes mellitus were independent risk factors for the development of
AP following RSI in the ED. The role of ET aspirate pepsin levels to predict the occurrence of AP did not yield promising results.

\section{Research Quality and Ethics Statement}

The authors of this manuscript declare that this scientific work complies with reporting quality, formatting, and reproducibility guidelines set forth by the EQUATOR Network. The authors also attest that this clinical investigation was determined to require Institutional Review Board/Ethics Committee review, and the corresponding protocol/approval number is IRB Min no: 11919 dated March 3, 2019. We also certify that we have not plagiarized the contents in this submission and have done a plagiarism check.

\section{OrCID}

Ramgopal Roshan (1) https://orcid.org/0000-0002-2799-3781 Sudhakar G Dhanapal @ https://orcid.org/0000-0001-8323-6075 Joshua Vijay ৫ https://orcid.org/0000-0002-9501-4995 Mamta Madhiyazhagan (1) https://orcid.org/0000-0001-9163-6212 Jayakumar Amirtharaj (1) https://orcid.org/0000-0001-6788-6879 Priya Ganesan ๑ https://orchid.org/0000-0001-6542-3766 Kundavaram PPAbhilash (1) https://orcid.org/0000-0002-2382-4411

\section{References}

1. Jain A, Sase NN, Mathew AR, Paul IJ, Abhilash Kundavaram PP, Ganesan P. Last breath in the emergency department. J Emerg Trauma Shock 2019;12(4):263-267. DOI: 10.4103/JETS.JETS_147_18.

2. Stevenson AGM, Graham CA, Hall R, Korsah P, McGuffie AC. Tracheal intubation in the emergency department: the Scottish district hospital perspective. Emerg Med J 2007;24(6):394-397. DOI: 10.1136/emj.2006.041988.

3. Inoue A, Okamoto H, Hifumi T, Goto T, Hagiwara $\mathrm{Y}$, Watase $\mathrm{H}$, et al. The incidence of post-intubation hypertension and association with repeated intubation attempts in the emergency department. PLOS ONE 2019;14(2):e0212170. DOI: 10.1371/journal.pone. 0212170.

4. BrownCA,BairAE,PallinDJ,WallsRM.Techniques, success, and adverse events of emergency department adult intubations. Ann Emerg Med 2015;65(4):363-370.e1. DOI: 10.1016/j.annemergmed.2014. 10.036 .

5. Alkhouri H, Vassiliadis J, Murray M, Mackenzie J, Tzannes A, McCarthy S, et al. Emergency airway management in Australian and New Zealand 
emergency departments: a multicentre descriptive study of 3710 emergency intubations. Emerg Med Australas 2017;29(5):499-508. DOI: 10.1111/1742-6723.12815.

6. Goto Y, Goto T, Hagiwara Y, Tsugawa Y, Watase H, Okamoto H, et al. Techniques and outcomes of emergency airway management in Japan: an analysis of two multicentre prospective observational studies, 2010-2016. Resuscitation 2017;114:14-20. DOI: 10.1016/j. resuscitation.2017.02.009.

7. Dunham MC, Barraco RD, Clark DE, Daley BJ, Davis FE 3rd, Gibbs MA, et al. Guidelines for emergency tracheal intubation immediately after traumatic injury. J Trauma 2003;55(1):162-179. DOI: 10.1097/01. ta.0000083335.93868.2c.

8. Marik PE. Aspiration pneumonitis and aspiration pneumonia. N Engl J Med 2001;344(9):665-671. DOI: 10.1056/NEJM200103013440908.

9. Gómez J, Baños V, Ruiz Gómez J, Soto MC, Muñoz L, Nuñez ML, et al. Prospective study of epidemiology and prognostic factors in community-acquired pneumonia. Eur J Clin Microbiol Infect Dis 1996;15(7):556-560. DOI: 10.1007/BF01709363.

10. Bartlett JG, Gorbach SL, Finegold SM. The bacteriology of aspiration pneumonia. Am J Med 1974;56(2):202-207. DOI: 10.1016/00029343(74)90598-1.

11. Ufberg JW, Bushra JS, Patel D, Wong E, Karras DJ, Kueppers F. A new pepsin assay to detect pulmonary aspiration of gastric contents among newly intubated patients. Am J Emerg Med 2004;22(7):612614. DOI: 10.1016/j.ajem.2004.08.019.

12. Martin LD, Mhyre JM, Shanks AM, Tremper KK, Kheterpal S. 3,423 emergency tracheal intubations at a university hospitalairway outcomes and complications. Anesthesiology 2011;114(1):42-48. DOI: 10.1097/ALN.0b013e318201c415.

13. Driver BE, Klein LR, Schick AL, Prekker ME, Reardon RF, Miner JR. The occurrence of aspiration pneumonia after emergency endotracheal intubation. Am J Emerg Med 2018;36(2):193-196. DOI: 10.1016/j. ajem.2017.07.066.

14. Lindenauer PK, Strait KM, Grady JN, Ngo CK, Parisi ML, Metersky M, et al. Variation in the diagnosis of aspiration pneumonia and association with hospital pneumonia outcomes. Ann Am Thorac Soc 2018;15(5):562-569. DOI: 10.1513/AnnalsATS.201709-728OC.
15. Schwartz DE, Matthay MA, Cohen NH. Death and other complications of emergency airway management in critically ill adults. A prospective investigation of 297 tracheal intubations. Anesthesiology 1995;82(2):367-376. DOI: 10.1097/00000542199502000-00007.

16. Oswalt JL, Hedges JR, Soifer BE, Lowe DK. Analysis of trauma intubations. Am J Emerg Med 1992;10(6):511-514. DOI: 10.1016/07356757(92)90173-U.

17. Thibodeau LG, Verdile VP, Bartfield JM. Incidence of aspiration after urgent intubation. Am J Emerg Med 1997;15(6):562-565. DOI: 10.1016/S0735-6757(97)90157-1.

18. Ja $R, D h L, B j T$, Jr RB. The value of intubating and paralyzing patients with suspected head injury in the emergency department. JTrauma 1991;31(3):371-375. DOI: 10.1097/00005373-19910300000010.

19. Rashkin MC, Davis T. Acute complications of endotracheal intubation: relationship to reintubation, route, urgency, and duration. Chest 1986;89(2):165-167. DOI: 10.1378/chest.89.2.165.

20. Taryle DA, Chandler JE, Good JT, Potts DE, Sahn SA. Emergency room intubations - complications and survival. Chest 1979;75(5):541-543. DOI: 10.1378/chest.75.5.541.

21. Lanspa MJ, Jones BE, Brown SM, Dean NC. Mortality, morbidity, and disease severity of patients with aspiration pneumonia. J Hosp Med 2013;8(2):83-90. DOI: 10.1002/jhm.1996.

22. MortTC. Emergency tracheal intubation: complications associated with repeated laryngoscopic attempts. Anesth Analg 2004;99(2):607-613. DOI: 10.1213/01.ANE.0000122825.04923.15.

23. Ufberg JW, Bushra JS, Karras DJ, Satz WA, Kueppers F. Aspiration of gastric contents: association with prehospital intubation. Am J Emerg Med 2005;23(3):379-382. DOI: 10.1016/j.ajem.2005.02.005.

24. Krishnan U, Mitchell JD, Messina I, Day AS, Bohane TD. Assay of tracheal pepsin as a marker of reflux aspiration. J Pediatr Gastroenterol Nutr 2002;35(3):303-308. DOI: 10.1097/00005176200209000-00012.

25. Metheny NA, Chang YH, Ye JS, Edwards SJ, Defer J, Dahms TE, et al. Pepsin as a marker for pulmonary aspiration. Am J Crit Care 2002;11(2):150-154. DOI: 10.4037/ajcc2002.11.2.150. 